\title{
A Remark on the Uniform Convergence of Some Sequences of Functions
}

\author{
Guy Degla1,2 \\ ${ }^{1}$ Institut de Mathematiques et de Sciences Physiques (IMSP), Porto-Novo, Benin \\ ${ }^{2}$ International Centre for Theoretical Physics (ICTP), Trieste, Italy \\ Email: gadegla@yahoo.fr \\ Received 12 May 2015; accepted 3 July 2015; published 6 July 2015 \\ Copyright (c) 2015 by author and Scientific Research Publishing Inc. \\ This work is licensed under the Creative Commons Attribution International License (CC BY). \\ http://creativecommons.org/licenses/by/4.0/ \\ (c) (i) Open Access
}

\begin{abstract}
We stress a basic criterion that shows in a simple way how a sequence of real-valued functions can converge uniformly when it is more or less evident that the sequence converges uniformly away from a finite number of points of the closure of its domain. For functions of a real variable, unlike in most classical textbooks our criterion avoids the search of extrema (by differential calculus) of their general term.
\end{abstract}

\section{Keywords}

\section{Sequence of Functions, Uniform Convergence, Metric, Boundedness}

\section{Introduction}

Let $X$ be a nonempty set, $f: X \rightarrow \mathbb{R}$ be a function and $\left\{f_{n}\right\}_{n \in \mathbb{N}}$ be a sequence of real-valued functions from $X$ into $\mathbb{R}$. Recall [1]-[3] that the sequence $\left\{f_{n}\right\}_{n \in \mathbb{N}}$ is said to converge uniformly to $f$ on $X$, if

$$
\lim _{n \rightarrow+\infty}\left(\sup \left\{\left|f_{n}(x)-f(x)\right|: x \in X\right\}\right)=0 .
$$

Obviously, if $\left\{f_{n}\right\}_{n \in \mathbb{N}}$ converges uniformly to $f$ on $X$, then for each $x \in X$ fixed, the sequence $\left\{f_{n}(x)\right\}_{n \in \mathbb{N}}$ converges to $f(x)$; that is, $\left\{f_{n}\right\}_{n \in \mathbb{N}}$ converges pointwise to $f$. It is also obvious that when $X$ is finite and $\left\{f_{n}\right\}_{n \in \mathbb{N}}$ converges pointwise to $f$ on $X$, then $\left\{f_{n}\right\}_{n \in \mathbb{N}}$ converges uniformly to $f$ on $X$. However this converse doesn't hold in general for an arbitrary (infinite) set $X$; i.e., the pointwise convergence may not imply the uniform convergence when $X$ is an arbitrary (infinite) set. 
One can observe that in the mathematical literature, there are very few known results that give conditions under which a pointwise convergence implies the uniform convergence. Concerning sequences of continuous functions defined on a compact set, we have the following facts:

Proposition A. (Dini's Theorem) [4]

If $K$ is a compact metric space, $f: K \rightarrow \mathbb{R}$ a continuous function, and $\left\{f_{n}\right\}_{n \in \mathbb{N}}$ a monotone sequence of continuous functions from $K$ into $\mathbb{R}$ that converges pointwise to $f$ on $K$, then $\left\{f_{n}\right\}_{n \in \mathbb{N}}$ converges uniformly to $f$ on $K$.

Proposition B. [5]

If $E$ is a Banach space and $\left\{T_{n}\right\}_{n \in \mathbb{N}}$ is a sequence of bounded linear operators of $E$ that converges pointwise to a bounded linear operator $T$ of $E$, then for every compact set $K \subset E,\left\{T_{n}\right\}_{n \in \mathbb{N}}$ converges uniformly to $T$ on K.

(For the sake of completeness, we give the proof of this proposition in the Appendix Section).

Therefore our aim is to highlight a new basic criterion that shows in some way how a sequence of real-valued functions can converge uniformly when it is more or less obvious that the sequence converges uniformly away from a finite number of points of the closure of its domain. In the case of sequences of functions of a real variable, our criterion avoids, unlike in most classical textbooks [3] [6], the search of extrema (by differential calculus) of their general terms. Several examples that satisfy the criterion are given.

\section{The Main Result (Remark)}

\subsection{Theorem}

Let $(E, d)$ be a metric space and $\Omega \neq \varnothing$ be a subset of $E$. Consider a sequence $\left\{f_{n}\right\}_{n \in \mathbb{N}}$ of functions defined from $\Omega$ to $\mathbb{R}$.

Suppose that there exists a function $f$ from $\Omega$ to $\mathbb{R}$, some points $a_{1}, \cdots, a_{k} \in \bar{\Omega}$, some positive real numbers $r_{1}, \cdots, r_{k}$ and a nonnegative constant $M$ such that

$$
\left|f_{n}(x)-f(x)\right| \leq M \prod_{i=1}^{k}\left[d\left(x, a_{i}\right)\right]^{r_{i}} ; \forall x \in \Omega \text { and for all } n \in \mathbb{N} .
$$

Suppose furthermore that for each $\varepsilon>0,\left\{f_{n}\right\}_{n \in \mathbb{N}}$ converges uniformly to $f$ on $\Omega \backslash \bigcup_{i=1}^{k} B\left(a_{i}, \varepsilon\right)$; where $B\left(a_{i}, \varepsilon\right)$ denotes the open ball of $E$ centered at $a_{i}$ and with radius $\varepsilon$.

Then the sequence of functions $\left\{f_{n}\right\}_{n \in \mathbb{N}}$ converges uniformly to $f$ on $\Omega$.

\section{Proof}

Let $\varepsilon>0$ be arbitrarily fixed (it may be sufficiently small in order to be meaningful). Then for every natural number $n$, we have

$$
\sup _{x \in \Omega}\left|f_{n}(x)-f(x)\right| \leq \max \left\{M \varepsilon^{r_{1}+\cdots+r_{k}}, \sup _{\substack{x \in \Omega, d\left(x, a_{i}\right) \geq \varepsilon ; i=1, \cdots, k}}\left|f_{n}(x)-f(x)\right|\right\} .
$$

Thus

$$
\limsup _{n \rightarrow+\infty}\left(\sup _{x \in \Omega}\left|f_{n}(x)-f(x)\right|\right) \leq M \varepsilon^{r_{1}+\cdots+r_{k}} \quad \forall \varepsilon>0
$$

by the uniform convergence of $\left\{f_{n}\right\}_{n}$ on $\Omega \backslash \bigcup_{i=1}^{k} B\left(a_{i}, \varepsilon\right)$.

And so

$$
\limsup _{n \rightarrow+\infty}\left(\sup _{x \in \Omega}\left|f_{n}(x)-f(x)\right|\right)=0
$$

i.e.,

$$
\lim _{n \rightarrow+\infty}\left(\sup _{x \in \Omega}\left|f_{n}(x)-f(x)\right|\right)=0
$$




\subsection{Observation}

The boundedness condition (D) of the above theorem can not be removed as shown by the sequence of functions defined from $[0,1]$ into $\mathbb{R}$ as follows:

$$
f_{n}(x)=n x(1-x)^{n} ; \quad x \in[0,1] \subset \mathbb{R} ;
$$

where $\mathbb{R}$ is equipped with its standard metric. Indeed, $\left\{f_{n}\right\}_{n \in \mathbb{N}}$ converges uniformly to 0 on $[\varepsilon, 1]$ for each $\varepsilon \in(0,1)$, but with $k=1$ and $a_{1}=0$ there is no positive number $r$ for which the condition (D) is satisfied since

$$
\forall r>0, \quad \sup _{n \in \mathbb{N}}\left(\sup _{0<x \leq 1} \frac{\left|f_{n}(x)\right|}{|x|^{r}}\right)=\infty .
$$

And we can see that $\left\{f_{n}\right\}_{n \in \mathbb{N}}$ does not converge uniformly to 0 on $[0,1]$ since

$$
\lim _{n \rightarrow \infty}\left(\sup _{0 \leq x \leq 1}\left|f_{n}(x)\right|\right)=\lim _{n \rightarrow \infty}\left(\frac{n}{n+1}\right)^{n+1}=\frac{1}{\mathrm{e}} \neq 0
$$

\section{Examples}

We give some examples that illustrate the theorem.

(1) Let $(E, d)$ be an infinite metric space and let $a \in E$ be fixed. Denote by $\varphi$ the function defined from $E$ into $\mathbb{R}$ by

$$
\varphi(x)=d(x, a), \quad \forall x \in E .
$$

Then the sequence of functions $\left\{\varphi_{n}\right\}_{n \in \mathbb{N}}$ defined by

$$
\varphi_{n}(x)=\frac{n[d(x, a)]^{2}+d(x, a)-\min \{1, d(x, a)\}}{1+n d(x, a)}, \quad \forall x \in E,
$$

converges uniformly to $\varphi$ on $E$.

(2) Given an infinite metric space $(E, d), a \in E$ and $\alpha \in(0,+\infty)$, we have that

i) the sequence of functions $\left\{u_{n}\right\}_{n \in \mathbb{N}}$ defined by

$$
u_{n}(x)=\frac{[d(x, a)]^{\alpha}}{[1+d(x, a)]^{n}} \quad \forall x \in E,
$$

converges uniformly to 0 on $E$,

ii) the sequence of functions $\left\{v_{n}\right\}_{n \in \mathbb{N}}$ defined by

$$
v_{n}(x)=[d(x, a)]^{\alpha} \exp (-n d(x, a)) \quad \forall x \in E,
$$

converges uniformly to 0 on $E$.

(3) Let $(E, d)$ be an infinite metric space and $\Omega$ be a bounded and infinite subset of $E$, let $a$ and $b$ be two different points of $\bar{\Omega}$ and let $\alpha$ and $\beta$ be two fixed positive numbers.

i) Consider the sequence of functions $\left\{f_{n}\right\}_{n \in \mathbb{N}}$ defined by

$$
f_{n}(x)=\frac{[d(x, a)]^{\alpha}[d(x, b)]^{\beta}}{1+n d(x, a) d(x, b)}, \quad x \in \Omega .
$$

Then $\left\{f_{n}\right\}_{n \in \mathbb{N}}$ converges uniformly to 0 on $\Omega$.

ii) Consider the sequence of functions $\left\{g_{n}\right\}_{n \in \mathbb{N}}$ defined by 


$$
g_{n}(x)=\frac{[d(x, a)]^{\alpha}[d(x, b)]^{\beta}}{[1+d(x, a) d(x, b)]^{n}}, \quad x \in \Omega .
$$

Then $\left\{g_{n}\right\}_{n \in \mathbb{N}}$ converges uniformly to 0 on $\Omega$.

iii) Consider the sequence of functions $\left\{h_{n}\right\}_{n \in \mathbb{N}}$ defined by

$$
h_{n}(x)=[d(x, a)]^{\alpha}[d(x, b)]^{\beta} \exp (-n d(x, a) d(x, b)), \quad x \in \Omega .
$$

Then $\left\{h_{n}\right\}_{n \in \mathbb{N}}$ converges uniformly to 0 on $\Omega$.

(4) In real analysis, we can recover the facts that each of the following sequences converges uniformly to 0 on their respective domains:

$$
\begin{aligned}
& (1-x) x^{n} ; \quad 0 \leq x \leq 1, \quad n=1,2,3, \cdots . \quad(1-x)^{n} \sqrt{x} ; \quad 0 \leq x \leq 1, \quad n=1,2,3, \cdots . \\
& \sin ^{n} x \cos x ; \quad 0 \leq x \leq \frac{\pi}{2}, \quad n=1,2,3, \cdots \quad \cos ^{n} x \sin x ; \quad 0 \leq x \leq \frac{\pi}{2}, \quad n=1,2,3, \cdots \text {. } \\
& x \mathrm{e}^{-n x} ; \quad x \geq 0, \quad n=1,2,3, \cdots .
\end{aligned}
$$

\section{Justifications (Proofs) of the examples}

(1) For every $n \in \mathbb{N}$, we have

$$
\left|\varphi_{n}(x)-\varphi(x)\right|=\frac{\min \{1, d(x, a)\}}{1+n d(x, a)}, \quad \forall x \in E .
$$

Therefore, on the one hand, for each $\varepsilon>0$, we have

$$
\left|\varphi_{n}(x)-\varphi(x)\right| \leq \frac{1}{1+n \varepsilon}, \quad \forall x \in E \backslash B(a, \varepsilon), \quad \forall n \in \mathbb{N},
$$

showing that $\left\{\varphi_{n}\right\}_{n \in \mathbb{N}}$ converges uniformly to $\varphi$ on $E \backslash B(a, \varepsilon)$.

On the other hand, we have

$$
\left|\varphi_{n}(x)-\varphi(x)\right| \leq d(x, a), \quad \forall x \in E \text { and } \forall n \in \mathbb{N}
$$

fulfilling condition (D) of the above theorem.

Thus $\left\{\varphi_{n}\right\}_{n \in \mathbb{N}}$ converges uniformly to $\varphi$ on $E$.

(2) i) On the one hand, for each $\varepsilon>0$, we have for all $x \in E \backslash B(a, \varepsilon)$ and for all $n \in \mathbb{N}$ with $n>\alpha$ :

$$
\left|u_{n}(x)\right|=u_{n}(x)=\frac{[d(x, a)]^{\alpha}}{[1+d(x, a)]^{\alpha}} \times \frac{1}{[1+d(x, a)]^{n-\alpha}} \leq \frac{1}{(1+\varepsilon)^{n-\alpha}}
$$

and so $\left\{u_{n}\right\}_{n \in \mathbb{N}}$ converges uniformly to 0 on $E \backslash B(a, \varepsilon)$.

On the other hand, we have

$$
\left|u_{n}(x)\right| \leq[d(x, a)]^{\alpha}, \quad \forall x \in E \text { and } \forall n \in \mathbb{N}
$$

fulfilling condition (D) of the above theorem.

Thus $\left\{u_{n}\right\}_{n \in \mathbb{N}}$ converges uniformly to 0 on $E$.

ii) The uniform convergence of $\left\{v_{n}\right\}_{n \in \mathbb{N}}$, follows that of $\left\{u_{n}\right\}_{n \in \mathbb{N}}$ since

$$
0 \leq v_{n}(x) \leq u_{n}(x), \quad \forall x \in E \text { and } \forall n \in \mathbb{N} .
$$

Observe that the uniform convergence of $\left\{v_{n}\right\}_{n \in \mathbb{N}}$ could also be proved using directly the above theorem.

(3) Note that for all natural number $n$, we have

$$
0 \leq h_{n} \leq g_{n} \leq f_{n}
$$


because

$$
\mathrm{e}^{-n t} \leq \frac{1}{(1+t)^{n}} \leq \frac{1}{1+n t}, \quad \forall t \geq 0 \text { and } \forall n \in \mathbb{N}
$$

following from

$$
1+n t \leq(1+t)^{n} \leq \mathrm{e}^{n t}, \quad \forall t \geq 0 \text { and } \forall n \in \mathbb{N} .
$$

Therefore it suffices to prove that $\left\{f_{n}\right\}_{n \in \mathbb{N}}$ converges uniformly to 0 on $\Omega$, although each of these three sequences can be handled directly with the above theorem.

Let $\delta$ be the diameter of $\Omega$.

Then on the one hand, for each $\varepsilon>0$, we have for all $x \in \Omega \backslash(B(a, \varepsilon) \cup B(b, \varepsilon))$ and for all $n \in \mathbb{N}$ :

$$
\left|f_{n}(x)\right|=f_{n}(x)=\frac{[d(x, a)]^{\alpha}[d(x, b)]^{\beta}}{1+n d(x, a) d(x, b)} \leq \frac{\delta^{\alpha+\beta}}{1+n \varepsilon^{2}},
$$

and so $\left\{f_{n}\right\}_{n \in \mathbb{N}}$ converges uniformly to 0 on $\Omega \backslash(B(a, \varepsilon) \cup B(b, \varepsilon))$.

On the other hand, we have

$$
\left|f_{n}(x)\right| \leq[d(x, a)]^{\alpha}[d(x, b)]^{\beta}, \quad \forall x \in \Omega \text { and } \forall n \in \mathbb{N}
$$

showing condition (D) of the above theorem.

Thus $\left\{f_{n}\right\}_{n \in \mathbb{N}}$ converges uniformly to 0 on $\Omega$ and we are done.

(4) i) Let us set $\psi_{n}(x)=(1-x) x^{n} ; 0 \leq x \leq 1$, with $n=1,2,3, \cdots$.

On the one hand, we have for every $n \in \mathbb{N}$ :

$$
\left|\psi_{n}(x)\right| \leq|x||x-1| \quad \forall x \in[0,1] .
$$

On the other hand, we have for every $\varepsilon \in\left(0, \frac{1}{2}\right)$ :

$$
\left|\psi_{n}(x)\right| \leq(1-\varepsilon)^{n} \quad \forall x \in(\varepsilon, 1-\varepsilon), \quad \text { for all } n \in \mathbb{N},
$$

showing that $\left\{\psi_{n}\right\}_{n}$ converges uniformly to 0 on $(\varepsilon, 1-\varepsilon)$.

Therefore, by taking $E=\mathbb{R}, \Omega=[0,1], \quad a_{1}=0, \quad a_{2}=1, r_{1}=r_{2}=1$ and $M=1$, the above theorem implies that $\left\{\psi_{n}\right\}_{n}$ converges uniformly to 0 on $[0,1]$.

ii) For $\psi_{n}(x)=(1-x)^{n} \sqrt{x} ; 0 \leq x \leq 1$, with $n=1,2,3, \cdots$.

On the one hand, we have for every $n \in \mathbb{N}$ :

$$
\left|\psi_{n}(x)\right| \leq|x-1| \sqrt{x} \quad \forall x \in[0,1] .
$$

On the other hand, we have for every $\varepsilon \in\left(0, \frac{1}{2}\right)$ :

$$
\left|\psi_{n}(x)\right| \leq(1-\varepsilon)^{n} \quad \forall x \in(\varepsilon, 1-\varepsilon), \text { for all } n \in \mathbb{N},
$$

showing that $\left\{\psi_{n}\right\}_{n}$ converges uniformly to 0 on $(\varepsilon, 1-\varepsilon)$.

Therefore, by taking $E=\mathbb{R}, \Omega=[0,1], a_{1}=0, a_{2}=1,2 r_{1}=r_{2}=1$ and $M=1$, the above theorem implies that $\left\{\psi_{n}\right\}_{n}$ converges uniformly to 0 on $[0,1]$.

iii) For $\psi_{n}(x)=\sin ^{n} x \cos x ; 0 \leq x \leq \frac{\pi}{2}$ with $n=1,2,3, \cdots$.

On the one hand, we have for every $n \in \mathbb{N}$ : 


$$
\left|\psi_{n}(x)\right| \leq|x|\left|x-\frac{\pi}{2}\right| \quad \forall x \in\left[0, \frac{\pi}{2}\right]
$$

On the other hand, we have for every $\varepsilon \in\left(0, \frac{\pi}{4}\right)$ :

$$
\left|\psi_{n}(x)\right| \leq \sin ^{n}\left(\frac{\pi}{2}-\varepsilon\right)=\cos ^{n} \varepsilon \quad \forall x \in\left(\varepsilon, \frac{\pi}{2}-\varepsilon\right), \quad \text { for all } n \in \mathbb{N},
$$

showing that $\left\{\psi_{n}\right\}_{n}$ converges uniformly to 0 on $\left(\varepsilon, \frac{\pi}{2}-\varepsilon\right)$ since $|\cos \varepsilon|<1$.

Therefore, by taking $E=\mathbb{R}, \Omega=\left[0, \frac{\pi}{2}\right], a_{1}=0, a_{2}=\frac{\pi}{2}, r_{1}=r_{2}=1$ and $M=1$, the above theorem implies that $\left\{\psi_{n}\right\}_{n}$ converges uniformly to 0 on $\left[0, \frac{\pi}{2}\right]$.

iv) For $\psi_{n}(x)=\cos ^{n} x \sin x ; 0 \leq x \leq \frac{\pi}{2}$ with $n=1,2,3, \cdots$.

On the one hand, we have for every $n \in \mathbb{N}$ :

$$
\left|\psi_{n}(x)\right| \leq|x|\left|x-\frac{\pi}{2}\right| \quad \forall x \in\left[0, \frac{\pi}{2}\right] .
$$

On the other hand, we have for every $\varepsilon \in\left(0, \frac{\pi}{4}\right)$ :

$$
\left|\psi_{n}(x)\right| \leq \cos ^{n} \varepsilon \quad \forall x \in\left(\varepsilon, \frac{\pi}{2}-\varepsilon\right), \quad \text { for all } n \in \mathbb{N},
$$

showing that $\left\{\psi_{n}\right\}_{n}$ converges uniformly to 0 on $\left(\varepsilon, \frac{\pi}{2}-\varepsilon\right)$ since $|\cos \varepsilon|<1$.

Therefore, by taking $E=\mathbb{R}, \Omega=\left[0, \frac{\pi}{2}\right], a_{1}=0, a_{2}=\frac{\pi}{2}, r_{1}=r_{2}=1$ and $M=1$, the above theorem implies that $\left\{\psi_{n}\right\}_{n}$ converges uniformly to 0 on $\left[0, \frac{\pi}{2}\right]$.

v) The example of $\psi_{n}(x)=x \mathrm{e}^{-n x} ; x \geq 0$, with $n=1,2,3, \cdots$, is a particular case of Example (2)-ii) above with $E=\mathbb{R}_{+}, d(x, y)=|x-y|$ for all $x, y \in \mathbb{R}_{+}, a=0$ and $\alpha=1$.

\section{References}

[1] Godement, R. (2004) Analysis I. Convergence, Elementary Functions. Springer, Berlin.

[2] Munkres, J. (2000) Topology. 2nd Edition. Printice Hall, Inc., Upper Saddle River.

[3] Ross, K.A. (2013) Elementary Analysis. The Theory of Calculus. Springer, New York. http://dx.doi.org/10.1007/978-1-4614-6271-2

[4] Godement, R. and Spain, P. (2005) Analysis II: Differential and Integral Calculus, Fourier Series, Holomorphic Fnctions. Springer, Berlin.

[5] Ezzinbi, K., Degla, G. and Ndambomve, P. (in Press) Controllability for Some Partial Functional Integrodifferential Equations with Nonlocal Conditions in Banach Spaces. Discussiones Mathematicae Differential Inclusions Control and Optimization.

[6] Freslon, J., Poineau, J., Fredon, D. and Morin, C. (2010) Mathématiques. Exercices Incontournables MP. Dunod, Paris. 


\section{Appendix}

In this section, we prove Proposition B for the sake of completeness.

Proof of Proposition B

Let $\varepsilon>0$ be given. By the Uniform Boundedness Principle, we have that $\sup _{n \geq 1}\left\|T_{n}\right\|<\infty$. So let $M=\sup _{n \geq 1}\left\|T_{n}\right\|$. Then there exist $a_{1}, a_{2}, \cdots, a_{m}$ such that $K \subset \bigcup_{i=1}^{m} B\left(a_{i}, \frac{\varepsilon}{2(M+1)}\right)$.

Also, $\forall x \in K, \exists j \in\{1, \cdots, m\}: x \in B\left(a_{j}, \frac{\varepsilon}{2(M+1)}\right)$. We have that

$$
\begin{aligned}
\left\|T_{n}(x)-T(x)\right\| & \leq\left\|T_{n}(x)-T_{n}\left(a_{j}\right)\right\|+\left\|T_{n}\left(a_{j}\right)-T\left(a_{j}\right)\right\|+\left\|T\left(a_{j}\right)-T(x)\right\| \\
& \leq\left\|T_{n}\right\|\left\|x-a_{j}\right\|+\|T\|\left\|a_{j}-x\right\|+\left\|T_{n}\left(a_{j}\right)-T\left(a_{j}\right)\right\| \\
& \leq \frac{\varepsilon M}{M+1}+\left\|T_{n}\left(a_{j}\right)-T\left(a_{j}\right)\right\| .
\end{aligned}
$$

It follows that $\sup _{x \in K}\left\|T_{n}(x)-T(x)\right\| \leq \varepsilon+\max _{1 \leq i \leq m}\left\|T_{n}\left(a_{i}\right)-T\left(a_{i}\right)\right\|$ and therefore

$$
\sup _{x \in K}\left\|T_{n}(x)-T(x)\right\| \rightarrow 0 \text {, as } n \rightarrow+\infty \text {. }
$$

